\title{
Asymptotic stability for coupled modal sliding mode control of vibration in a flexible structure
}

\author{
Ming-Chang Pai ${ }^{\mathrm{a}, *}$ and Alok Sinha ${ }^{\mathrm{b}}$ \\ ${ }^{a}$ Department of Automation Engineering, Nan Kai Institute of Technology, Tsa-Tun, Nantou, 54210, Taiwan \\ ${ }^{\mathrm{b}}$ Department of Mechanical and Nuclear Engineering, The Pennsylvania State University, University Park, PA \\ 16802, USA
}

Received 1 May 2007

Revised 2008

\begin{abstract}
This paper deals with the asymptotic stability of the coupled modal sliding mode control of a vibratory structure in the presence of parametric uncertainties. The method is based on the linear fractional transformation and the small gain theorem / $\mu$ analysis. Using a flexible tetrahedral truss structure, a numerical example is presented to verify the theoretical analysis.
\end{abstract}

Keywords: Coupled modal space control, sliding mode control, linear fractional transformation, small gain theorem, $\mu$ analysis

\section{Introduction}

An effective approach to robust control is the sliding mode control theory [1-5]. The main characteristic of a sliding mode control system is the non-linear control action which forces the state trajectories of the system to reach the intersection of a desired set of sliding hyperplanes (sliding manifold) containing the origin of the state space in a finite time and to stay on that sliding manifold. The main advantage of the method is that the closed-loop system can be designed to be robust with respect to parametric uncertainties and external disturbances, provided that their bounds are known and certain invariance conditions or matching conditions are satisfied [2]. Kao and Sinha [3] used coupled modal space control (CMSC) in conjunction with the sliding mode control to attenuate vibration in a flexible structure. They have studied the asymptotic behavior of closed-loop systems inside boundary layers for a full-state feedback and deterministic control of vibration in a flexible structure with uncertain parameters which do not satisfy matching conditions [2]. Inside boundary layers, they have found three types of steady state solutions: zero, non-zero constant and limit cycle. However, they did not show how the known parameters' bounds can be used to ensure that the system reaches the zero solution which is the origin of the state space inside boundary layers. In this paper, the small gain theorem / $\mu$ analysis has been applied to obtain the bounds of uncertainties so that the robust stability or zero vibration of a flexible structure will be achieved. Using a flexible tetrahedral truss structure, a numerical example is presented to verify the theoretical analysis.

\footnotetext{
${ }^{*}$ Corresponding author. Tel.: +886 492563489 3308; E-mail: pmc@nkc.edu.tw.
} 


\section{Sliding mode control of a flexible structure with parametric uncertainties}

Consider the dynamics of a flexible structure with $k$ uncertain parameters, $\delta_{o 1}, \cdots, \delta_{o k}$, and which are expressed as

$$
\dot{x}(t)=A x(t)+B u(t)
$$

or

$$
\dot{x}(t)=\left(\bar{A}+\sum_{i=1}^{k} \delta_{o i} \hat{A}_{i}\right) x(t)+B u(t)
$$

where states $x(t) \in R^{n}$, input $u(t) \in R^{m}$, and the nominal system is given by known matrices $\bar{A}$ and $B$. The parametric uncertainties in the nominal system are reflected by the $k$ scalar uncertain real parameters $\delta_{o 1}, \cdots, \delta_{o k}$, which can be positive or negative. The knowledge about the structure of the uncertainty is contained in the matrix $\hat{A}_{i}$, which indicate how the $i^{t h}$ uncertainty, $\delta_{o i}$, affects the state space model. Assume that the bound of the uncertain matrix $\Delta A$ is $Q$, i.e.,

$$
\Delta A=A-\bar{A} \text { and }|\Delta A|=\left|\sum_{i=1}^{k} \delta_{o i} \hat{A}_{i}\right| \leqslant Q, \quad Q>0
$$

where the absolute value of the matrix implies the matrix with the absolute value of each element and the inequality sign holds good for each element of the matrices. Also, the uncertain matrix $\Delta A$ need not satisfy the so-called matching conditions, i.e., $\Delta A=B D$, where $D$ is a bounded matrix [2]. The goal of this paper is to find the upper bound of $\delta_{o i}$, for the asymptotic stability of the closed-loop system.

It is assumed that $(\bar{A}, B)$ is controllable. For $m$ inputs, $m$ hyperplanes passing through the origin of the state space are defined as follows:

$$
s_{i}(t)=g_{i}^{T} x(t) \quad(i=1, \cdots, m) \text { or } S(t)=G x(t)
$$

where

$$
S(t)=\left[s_{1}(t), \cdots, s_{m}(t)\right]^{T}
$$

and

$$
G=\left[g_{1}, g_{2}, \cdots, g_{m}\right]^{T}
$$

To satisfy reaching conditions $s_{i}(t) \dot{s}_{i}(t)<0$ under parametric uncertainties and eliminate the chattering behavior, the control law [3] is derived to be

$$
u(t)=u_{e q}(t)-(G B)^{-1} \operatorname{diag}(E) \operatorname{sat}\left(\frac{S(t)}{\rho}\right)
$$

where

$$
u_{e q}(t)=-(G B)^{-1} G \bar{A} x(t)
$$

and $\operatorname{diag}(E)$ is a $m \times m$ diagonal matrix with the $i^{t h}$ diagonal element to be the $i^{\text {th }}$ element of a $m \times 1$ vector $e_{1}$, which is defined [3] as

$$
e_{1}=|G| Q|x(t)|+\eta_{1} \quad \eta_{1}>0
$$

The $i^{\text {th }}$ element of $\operatorname{sat}\left(\frac{S(t)}{\rho}\right)$ is described as

$$
\begin{aligned}
\operatorname{sat}\left(\frac{s_{i}(t)}{\rho_{i}}\right) & =\operatorname{sgn}\left(s_{i}(t)\right) \quad \text { if }\left|s_{i}(t)\right|>\rho_{i} \\
& =\frac{s_{i}(t)}{\rho_{i}} \quad \text { otherwise }
\end{aligned}
$$

where $\rho_{i}$ is a measure of the boundary layer thickness [1,3] around the $i^{\text {th }}$ hyperplane. 


\section{Robustness inside boundary layers}

The dynamic equations inside the boundary layers can be obtained by substituting Eq. (6) into Eq. (1a), and applying the definition of the saturation function in Eq. (9). The result is

$$
\dot{x}(t)=\Gamma x(t)-O(x(t))
$$

where

$$
\begin{aligned}
& \Gamma=A-B(G B)^{-1} G \bar{A}-B(G B)^{-1} \operatorname{diag}\left(\frac{\eta_{1}}{\rho}\right) G \\
& \mathrm{O}(x(t))=B(G B)^{-1} \operatorname{diag}(|G| Q|x(t)|) \operatorname{diag}\left(\frac{1}{\rho}\right) G x(t)
\end{aligned}
$$

The closed-loop system with uncertain system parameters will have a stable zero steady state if all of the eigenvalues of the linearized system matrix of Eq. (10) around the zero solution, i.e., the eigenvalues of $\Gamma$ are in the left half of the complex plane [3]. It should be noted that the matrix $\Gamma$ in Eq. (11) contains the matrix $A$ which has uncertain parameters. To examine the stability of the matrix $\Gamma$, the following system is considered:

$$
\dot{x}(t)=\Gamma x(t)=A x(t)-B K_{o 1} x(t)
$$

where

$$
K_{o 1}=\left[(G B)^{-1} G \bar{A}+(G B)^{-1} \operatorname{diag}\left(\frac{\eta_{1}}{\rho}\right) G\right]
$$

In order to utilize the small gain theorem / $\mu$ analysis, the system Eq. (13) will be transformed into the $M-\Delta_{o}-K_{o}$ diagram (Fig. 1) through the linear fractional transformation method [6, pp. 262-264]. Let

$$
M \triangleq\left[\begin{array}{cr}
\bar{A}+\sum_{i=1}^{k} \delta_{i} \hat{A}_{i} B \\
I_{n} & 0
\end{array}\right]
$$

and

$$
\delta_{i}\left[\begin{array}{cc}
\hat{A}_{i} & 0 \\
0 & 0
\end{array}\right]=\left[\begin{array}{c}
L_{o i} \\
W_{o i}
\end{array}\right]\left[\delta_{i}\right]\left[\begin{array}{c}
R_{o i} \\
Z_{o i}
\end{array}\right]^{*} \text { for each } i
$$

where $*$ denotes matrix transpose, $L_{o i} R_{o i}=\hat{A}_{i}, W_{o i}=0$ and $Z_{o i}=0$.

Using Eq. (14b), the matrix $M$ in Eq. (14a) can be written as

$$
M \triangleq\left[\begin{array}{ll}
\bar{A} & B \\
I_{n} & 0
\end{array}\right]+\left[\begin{array}{lll}
L_{o 1} & \cdots & L_{o k} \\
0 & \cdots & 0
\end{array}\right]\left[\begin{array}{lll}
\delta_{o 1} & & \\
& \ddots & \\
& & \delta_{o k}
\end{array}\right]\left[\begin{array}{lll}
R_{o 1} & \cdots & R_{o k} \\
0 & \cdots & 0
\end{array}\right]^{*}
$$

Hence, the matrices $M, K_{o}$ and $\Delta_{o}$ in Fig. 1. can be expressed [6, pp. 262-264] as follows

$$
M=\left[\begin{array}{ccc}
\operatorname{bar} A & B_{o} & B \\
C_{o} & 0 & 0 \\
I_{n} & 0 & 0
\end{array}\right] \text { and }\left[\begin{array}{l}
\dot{x}(t) \\
z_{o}(t) \\
y_{o}(t)
\end{array}\right]=M\left[\begin{array}{l}
x(t) \\
d_{o}(t) \\
u_{o}(t)
\end{array}\right]
$$

where

$$
\begin{aligned}
B_{o} & =\left[L_{o 1} \cdots L_{o k}\right] \\
C_{o} & =\left[R_{o 1} \cdots R_{o k}\right]^{*}
\end{aligned}
$$

and

$$
\Delta_{o}=\operatorname{diag}\left\{\delta_{o 1}, \cdots, \delta_{o k}\right\} .
$$




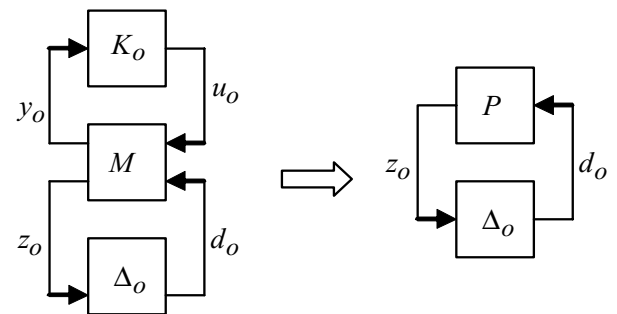

Fig. 1. $M-\Delta_{o}-K_{o}$ and $P-\Delta_{o}$ block diagrams.

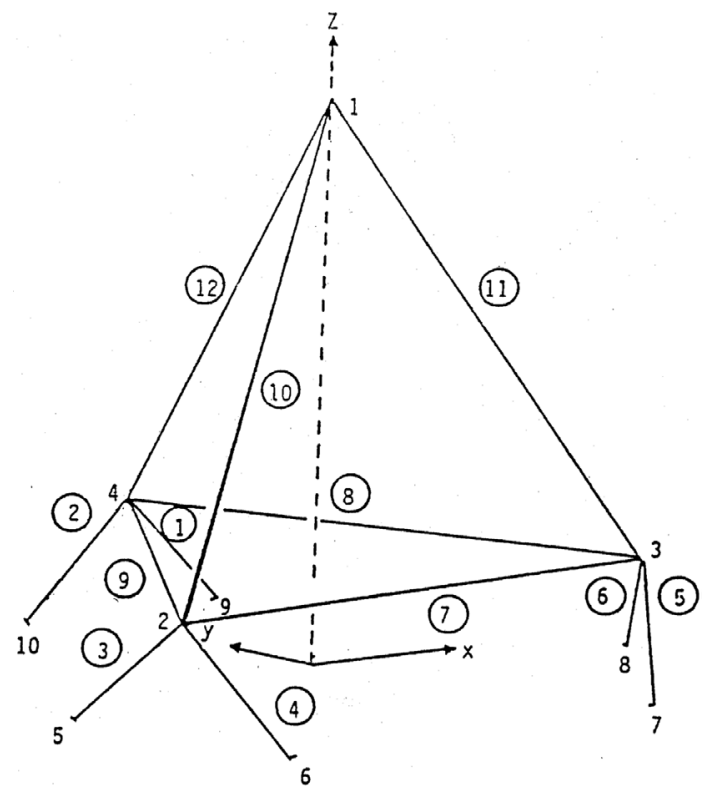

Fig. 2. A flexible tetrahedral structure [3].

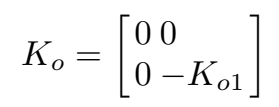

The transfer function $P(s)$ in Fig. 1 is defined as follows:

$$
P(s) \triangleq F_{u}\left(M(s), K_{o}(s)\right)
$$

where $F_{u}\left(M(s), K_{o}(s)\right)$ is the upper linear fractional transformation (LFT) [6].

Once the infinity-norm $\|P(s)\|_{\infty}$ is obtained, the small gain theorem / $\mu$ analysis [6] is applied. According to the small gain theorem, the condition for robust stability of the closed-loop system Eq. (13) is $\left\|\Delta_{o}\right\|_{\infty}<1 / \gamma$ if and only if $\|P(s)\|_{\infty}<\gamma$. On the basis of the $\mu$ analysis, the condition for robust stability of the closed-loop system Eq. (13) is $\left\|\Delta_{o}\right\|_{\infty} \leqslant 1 / \beta$ if and only if $\sup _{\omega \in R} \mu_{\Delta}(P(j \omega)) \leqslant \beta$. Therefore, the sliding mode control with uncertain system parameters will have a stable zero steady state solution if the norm of uncertainties is within the upper bound of parametric uncertainties, $1 / \gamma$ or $1 / \beta$, i.e.; all of eigenvalues of the matrix $\Gamma$ are in the left half of the complex plane.

\section{Illustrative example}

For the purpose of illustration, the tetrahedral truss structure, Fig. 2, which is the same model as used in the paper by Kao and Sinha [3], has been chosen. Three actuators located at elements 1-3 control the first four modes of the 
tetrahedral truss structure. For the first four controlled modes, the state space equations of the controlled modes system can be expressed as follows:

$$
\dot{x}(t)=\left(A+\sum_{j=1}^{8} \delta_{j} \mathrm{~A}_{j}\right) x(t)+B u(t)
$$

where $x(t)$ is the $8 \times 1$ controlled state vector and

$$
\begin{aligned}
A & =\operatorname{diag}\left(\left[\begin{array}{lll}
0 & 1 \\
-\bar{\omega}_{i}^{2}-2 \bar{\zeta}_{i} \bar{\omega}_{i}
\end{array}\right]\right) \quad(i=1, \cdots, 4) \\
B & =\left[\begin{array}{ccc}
0 & 0 & 0 \\
-0.023 & -0.067 & -0.044 \\
0 & 0 & 0 \\
-0.112 & 0.017 & 0.069 \\
0 & 0 & 0 \\
-0.077 & 0.271 & 0.046 \\
0 & 0 & 0 \\
0.189 & -0.06 & -0.249
\end{array}\right]
\end{aligned}
$$

Here, the estimated natural frequencies are $\bar{\omega}_{1}=1.342 \mathrm{rad} / \mathrm{s}, \bar{\omega}_{2}=1.643 \mathrm{rad} / \mathrm{s}, \bar{\omega}_{3}=2.891 \mathrm{rad} / \mathrm{s}$ and $\bar{\omega}_{4}=$ $2.958 \mathrm{rad} / \mathrm{s}$. The estimated damping coefficient $\bar{\zeta}_{i}$ is assumed to be 0.005 for each mode.

The relations among parametric uncertainties $\delta_{j}(j=1,3,5,7)$, actual natural frequencies $\omega_{i}$ and estimated natural frequencies $\bar{\omega}_{i}$ can be expressed as follows.

$$
\left|\omega_{i}^{2}-\bar{\omega}_{i}^{2}\right| \leqslant \delta_{j} \bar{\omega}_{i}^{2} \quad(j=2 i-1, \quad i=1,2,3,4)
$$

The uncertainties in $\zeta_{i}$ are defined as follows.

$$
\left|2 \zeta_{i} \omega_{i}-2 \bar{\zeta}_{i} \bar{\omega}_{i}\right| \leqslant \delta_{j}\left(2 \bar{\zeta}_{i} \bar{\omega}_{i}\right)(j=2 i, \quad i=1,2,3,4)
$$

The uncertainty matrix $\Delta_{o}$ in Eq. (19) with $k=8$ can be expressed as

$$
\Delta_{o}=\operatorname{diag}\left(\delta_{j}\right) \quad(j=1, \cdots, 8)
$$

The matrices $B_{o}$ and $C_{o}$ in Eqs (17) and (18) can be obtained by using Eqs (14b), (15), (23) and Eqs (25)-(27).

The $H_{\infty}$ full state feedback method [6] has been used to place the poles of the reduced-order system [3] with $S(t)=0$ and to obtain the sliding hyperplane matrix $G$. The value of $\rho$ is arbitrarily selected to be one and the value of $\eta_{1}$ is found to be 29.5886 by minimizing the infinity-norm $\|P(s)\|_{\infty}$ in Eq. (21). It shows that $\|P(s)\|_{\infty}=4.6772$ and the upper bound of the parametric uncertainty $\left\|\Delta_{o}\right\|_{\infty}=0.2138$ can be obtained by using the small gain theorem, and the $\sup _{\omega \in R} \mu_{\Delta}(P(j \omega))=2.8162$ and the upper bound of parametric uncertainty $\left\|\Delta_{o}\right\|_{\infty}=0.3551$ can be obtained by using the $\mu$ analysis. If the norm of uncertainties is within the upper bound of parametric uncertainties $\Delta_{o}$, all of eigenvalues of the closed-loop system Eq. (13) are in the left half of the complex plane and the sliding mode control with uncertain system parameters will have a stable zero steady state solution. As expected, the $\mu$ analysis gives a less conservative bound on uncertainties for robust stability.

Results from numerical integration of governing differential equations are generated for $\delta_{j}=0.15 \quad(j=$ $1, \cdots, 8)$, and shown in Figs 3 and 4 . This set of uncertainties corresponds to $\left\|\Delta_{o}\right\|_{\infty}=0.15$, which is less than the upper bound obtained from the $\mu$ analysis for sliding mode $/ H_{\infty}$ controller. In Fig. 3, sliding hyperplane variables $s_{i}(t)(i=1,2$, and 3$)$ are shown. Initially, the system is outside boundary layers. However, after a finite time, the system reaches inside boundary layers, and remains inside them. In Fig. 4, all controlled states are plotted. This plot indicates that the system reaches the origin of state space inside boundary layers. 


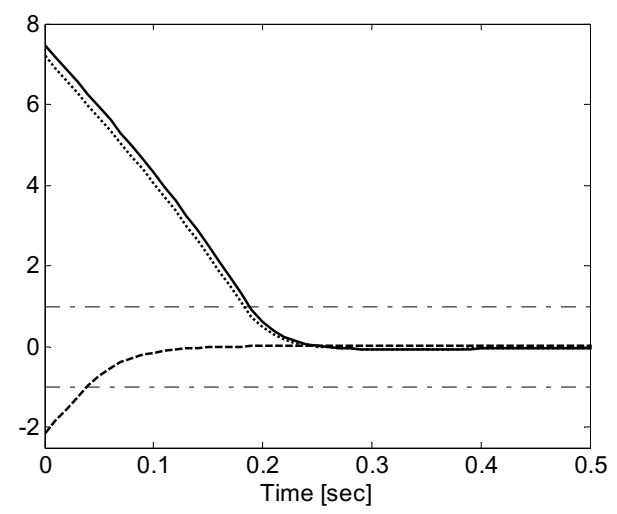

Fig. 3. Sliding hyperplane variables, $s_{i}(t)$, versus time. $---s_{1},-s_{2}, \cdots s_{3},-\cdot-\cdot$ boundary layer.
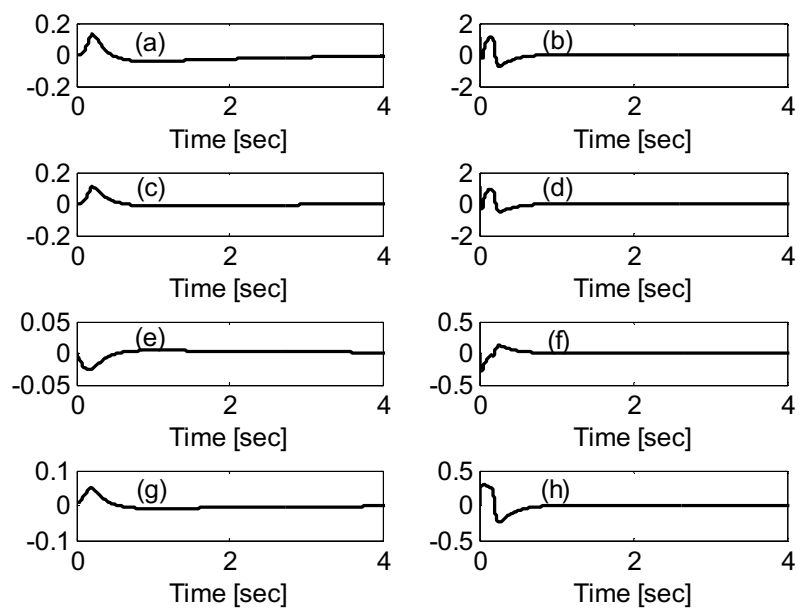

Fig. 4. Controlled states versus time $\left(\delta_{j}=0.15(j=1, \cdots, 8)\right.$ or $\left.\left\|\Delta_{o}\right\|_{\infty}=0.15\right)$ (a) $x_{1}$. (b) $x_{2}$. (c) $x_{3}$. (d) $x_{4}$. (e) $x_{5}$. (f) $x_{6}$. (g) $x_{7}$. (h) $x_{8}$.

\section{Conclusion}

For any suitable choice of sliding hyperplanes in the coupled modal vibration control, the bounds of parametric (natural frequencies and damping ratios) uncertainties have been obtained for the asymptotic stability (or zero vibration) of the closed-loop system. The parametric errors in the original system do not need to satisfy the so-called matching conditions or invariance conditions. The analysis utilizes linear fractional transformation and the small gain theorem / $\mu$ analysis.

\section{References}

[1] H. Asada and J.J.E. Slotine, Robot Analysis and Control, Wiley, New York, 1986.

[2] B. Drazenovic, The invariant condition in variable structure systems, Automatica 5 (1969), 287-295.

[3] C.K. Kao and A. Sinha, Coupled modal sliding mode control of vibration in flexible structures, AIAA Journal of Guidance, Dynamics and Control 15(1) (1992), 65-73.

[4] A. Sinha and C.K. Kao, Independent modal sliding mode control of vibration in flexible structures, Journal of Sound and Vibration 147(2) (1991), 352-358.

[5] V.I. Utkin, Variable structure systems: present and future, Automation and Remote Control 44(9) (1983), 1105-1119.

[6] K. Zhou, J.C. Doyle and K. Glover, Robust and Optimal Control, Prentice-Hall, Inc., 1996. 

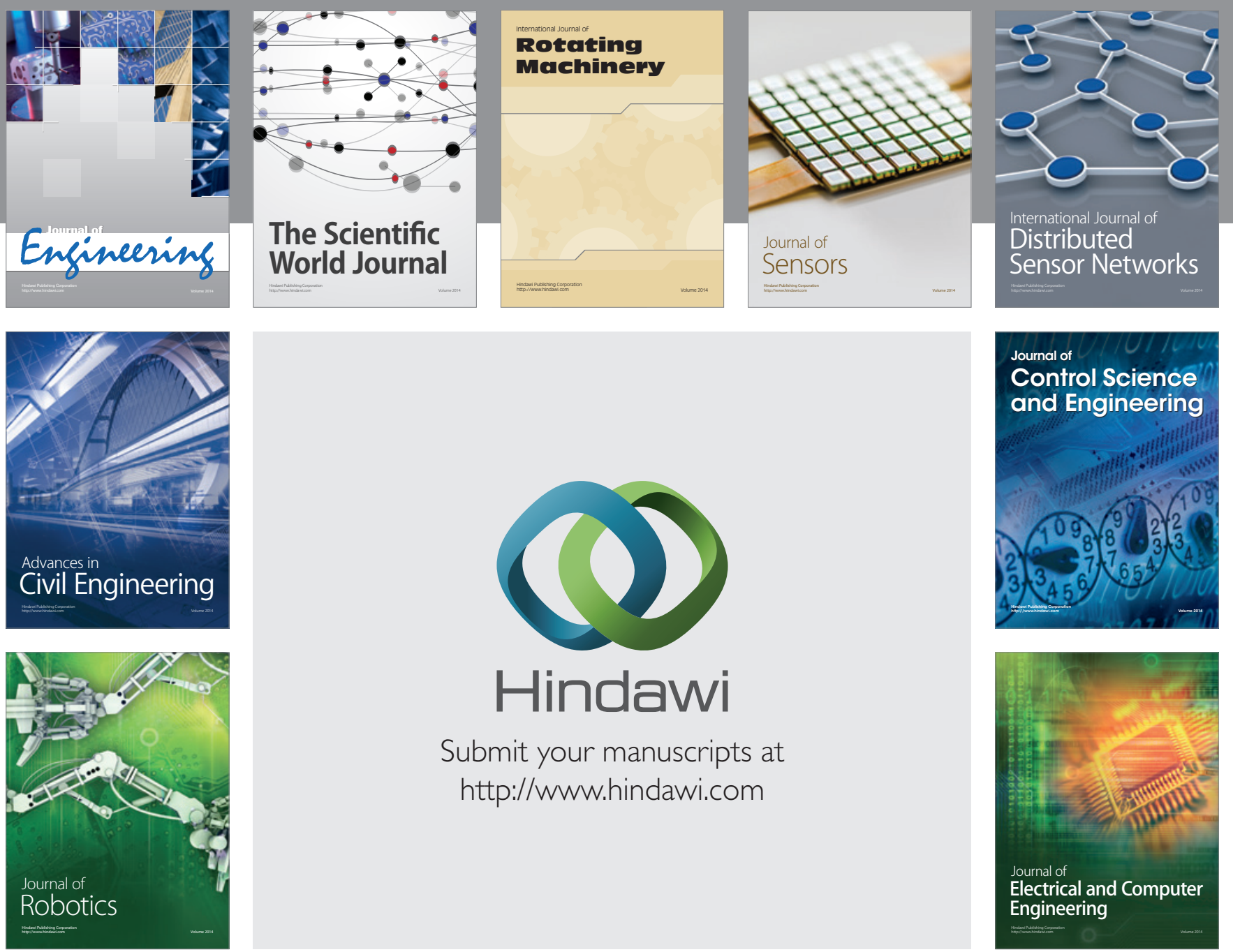

Submit your manuscripts at

http://www.hindawi.com
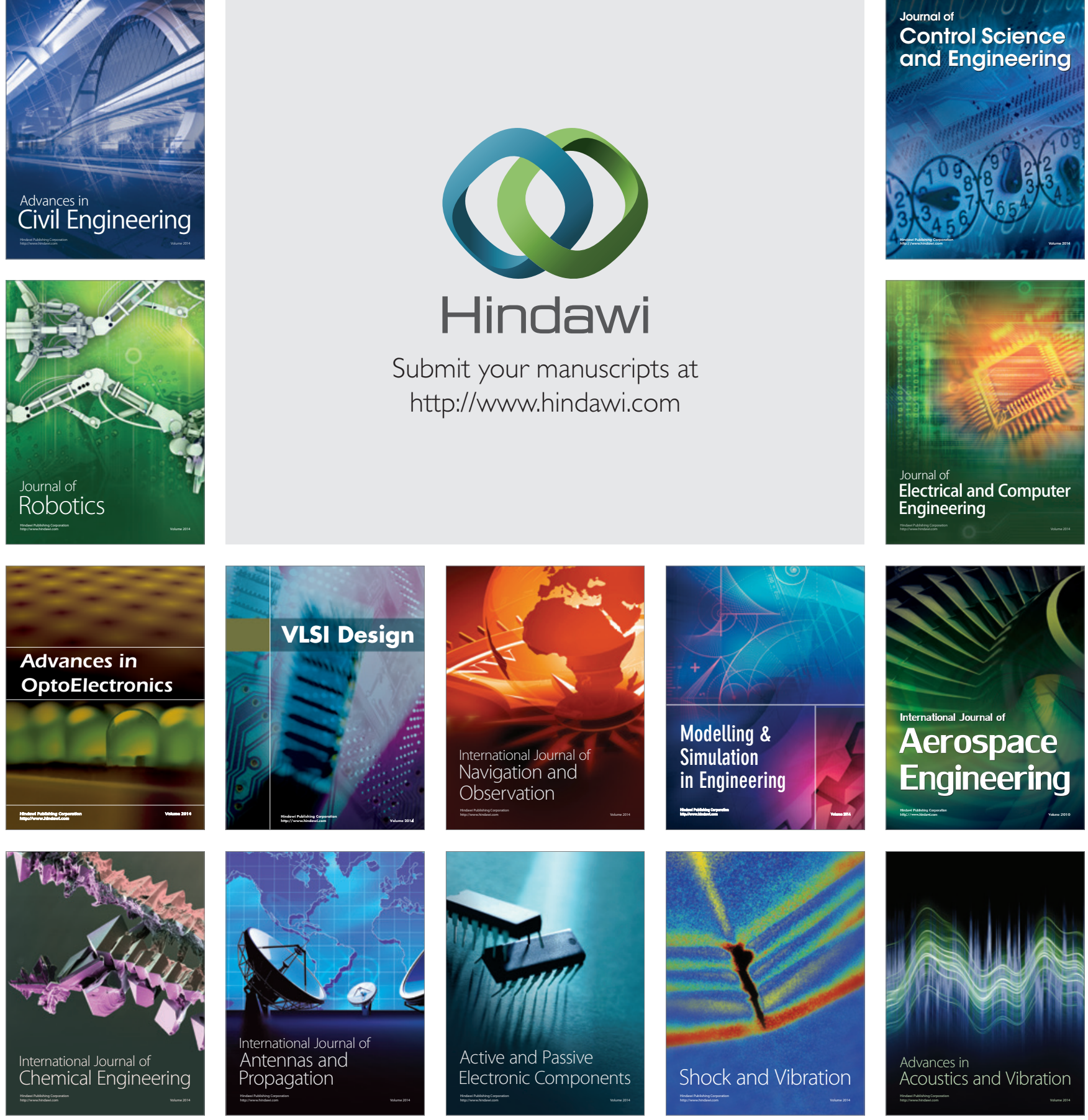